\title{
Big Data for Big Problems Climate Change, Water Availability, and Food Safety
}

\author{
Walter Armbruster \\ Farm Foundation (retired) \\ Oakbrook, IL, USA \\ walt@farmfoundation.org
}

\author{
Margaret MacDonell \\ Environmental Science Division, \\ Argonne National Laboratory, Argonne, IL, USA \\ macdonell@anl.gov
}

\begin{abstract}
Agriculture and the food supply chain face big problems related to climate change, water availability and food safety that challenge the ability to provide safe and affordable food security for the burgeoning world population. Applying data analytics to improve agricultural productivity involves a number of challenges in collecting, processing, and packaging data and findings in ways to provide practical tool for use by farmers and the food supply cahin. To meet the growing demand for agricultural products despite the impacts of climate change and the limited availability of additional agricultural lands, it will be necessary to adopt climate-smart agriculture. Developments in improving the capability of the agricultural sector to withstand the productivity and economic impacts of drought are becoming more critical and require capacity to monitor regional and national soil moisture content. A global drought monitoring framework is being developed by scientists to integrate continental and regional drought monitoring. Another critical element of food security is food safety throughout the food processing and distribution supply chain. Risk assessment and cost/benefit analysis can be used to evaluate pathogen interventions in the food supply chain, then used to set priorities and targets for specific foodborne illnesses. However, concerns about data ownership and control, especially affecting potential privacy issues, will likely need attention throughout the food supply chain. The informatics community is in a prime position to develop robust and productive approaches to helping assure that society improves worldwide food security by insightful application of big data.
\end{abstract}

\section{INTRODUCTION}

Agriculture and the food supply chain face big problems related to climate change, water availability and food safety that challenge the ability to provide safe and affordable food security for the burgeoning world population. While the United Nations Food and Agriculture Organization projects a 9 billion world population by 2050 , water scarcity is becoming an increasing concern for agricultural production and the food processing industry as the growing numbers of people put greater stress on the available water supply. Simultaneously, climate change is anticipated to result in more severe and lengthy droughts further reducing water availability. Shifting moisture patterns due to climate change put stress on some highly productive agricultural regions such as the western United States that rely on heavy mountain snow packs from winter to slowly melt and refurbish reservoirs during the warm seasons. This snowmelt provides farms with irrigation water, municipal water supplies, and environmentally sound river flows.
Further challenges to food security involve food safety as larger quantities of fresh produce and basic agricultural commodities are handled in the harvesting and processing segments of the industry. Higher field temperatures lead to some direct problems, such as potential increases in aflatoxin contamination of grains. Increased production in new areas or strains in handling larger volumes of production in traditional areas may increase food safety problems during the processing and distribution stages. In particular, fresh foods and especially fresh produce may be contaminated in the production fields, in harvesting, or in processing plants as well as further along the supply chain during transportation, in retail and food service institutions, and finally in the home.

The many challenges in the field production to consumer consumption supply chain sequence may be better addressed with the evolving application of big data analytics to monitor foods as they flow through the production, processing and food distribution systems. Big data here refers to the evolving term that describes voluminous amounts of structured, partly-structured and unstructured data that have the potential to be mined for information applicable to a specific concern or situation. For example, data analytics can help improve farm level productivity through precision agriculture, and facilitate climate smart agricultural solutions to deal with weather variability and drought impacts. Big data allows better monitoring of regional and national soil moisture content to plan and adapt food production practices to the extent possible, and at the other end of the supply chain to monitor and evaluate alternative solutions for and implement practices to reduce food safety incidents. Resulting information can support development and implementation of best practices to maximize production efficiency, minimize contamination in the handling and processing phases, assure safe transportation, and greatly reduce contamination in retail and food service institutions.

However, the analysis of these large data sets and resulting use of and access to the information derived from it creates some concerns about data ownership, access and privacy for individual business operations. The policy issues involved in the potential applications of big data to the agricultural and food industry to deliver food security over the longer term will be important to successful application 
of the strengths of big data to provide a sound basis for decisions of individual farmers and businesses. The paper addresses that topic after examining the benefits of data analytics for farm productivity, to facilitate climate smart agriculture, to allow adaptation to weather and drought challenges, and to strengthen supply chain food safety, all of which must be accomplished while respecting data privacy issues.

\section{DATA ANALYTICS FOR FARM PRODUCTIVITY}

Data technology has potential to create significant value through data driven farming to enable improved decision making at the individual producer level. Advanced data science models are needed to improve farm level decisions about timing of planting, fertilization needs, applications of chemicals for weed and pest control, irrigation timing, and overall operating decisions critical to obtaining higher yields and simultaneously lowering costs of inputs. Low cost sensors, cheap wireless technology, and other data collections tools for crop monitoring throughout the growing season have the potential to supplement the farmers innate knowledge of their land, but move to more scientific than art-based farm management. This is particularly important as farms increase in size and complexity to the extent that $85 \%$ of U.S. production now comes from the top $15 \%$ of farms by size [1].

Using data analytics to improve agricultural productivity involves a number of challenges in collecting, processing, and packaging data and findings in ways to provide practical tools for use by farmers and ranchers. Currently too much climate data lacks spatial and/or temporal resolution needed to localize recommendations, develop appropriate crop adaptations through research, and create policy incentives or private sector opportunities to address the issues involved. Further, the current sensors are not always error proof and thus data gaps or inaccuracies may be problematic. For example, to improve spatial and temporal data collection, sensors must be properly located and installed, as well as maintained and calibrated regularly [2]. This is but one of the technical and analytic challenges in making big data of significant value to individual agricultural producers.

Agricultural producers in developed countries in particular employ remote sensing and risk management tools in combination to create better informed decisions on a field by field basis. Increasingly sophisticated remote sensing components of agricultural equipment provide massive amounts of data about such site-specific factors as soil types, water holding capacity and saturation levels, chemical composition of the soil to determine whether additional fertilization is needed, and prevalence of disease or insect infestation in the crop. Ownership and control of the data generated about planting dates, pesticide applications, and crop yields by sensors built into agricultural equipment has been a point of contention between farmers using the equipment and the often large companies providing the equipment. These agribusinesses analyze the data to help farmers determine what seeds to plant, when to treat fields with herbicides or pesticides, and in planning harvest activities. This issue is addressed later in this paper.

A particular value of remote sensing is that it allows the harnessing of spatial and temporal dynamics within and among growing seasons. It allows detection of plant canopy parameters in the context of plant growth dynamics and facilitates evaluation of changes in crop yield response to weather variation. For example, combining up to more than 30 different vegetative indices allows estimation of plant canopy parameters to track drought progression across the United States by drought severity [3]. The benefit to farmers and markets for food and agricultural products is the potential to build into their planning horizon and risk reduction strategies the potential production and price impacts from weather variation.

A major problem currently is that climate data often lacks spatial or temporal resolution to the level useful to the farm operations. There is need to rapidly process data to deliver timely information readily useful to the producer. The Southeast Climate Consortium consists of eight universities located in the southeast United States which have come together to create AgroClimate to do just that. This approach to adapting agricultural production to climate change, involves a model developed with initial funding from the U.S. Department of Agriculture. AgroClimate provides a set of tools for managing climate risk in agriculture. The tools and information are created from realtime monitoring, as well as from short-term and seasonal forecasts. Such tools may be map-based, tied to specific weather stations, and vary in temporal resolution. Data generated in the model can be analyzed using readily available statistical techniques to translate climate and weather data/information into decision aids to help farmers reduce risk. By facilitating better decisions, producers should experience reduced production risk, increased profitability, and more sustainable farming systems. For example, disease alert systems such as the Strawberry Advisory System allows producers to optimize spray applications, applying fungicides only when conditions are favorable for disease development based on 15-minute temperature and leaf wetness data where weather monitoring stations are available. Another example is an app for irrigation scheduling changes, depending upon probability of rain in the next few hours, which can provide significant water savings. Similar AgroClimate initiatives are occurring in Brazil, Paraguay, and Mozambique [1].

In the private sector, the most high profile early attempt to harness big data and weather data to improve on-farm decision making involves Monsanto Corporation's purchase of The Climate Corporation (TCC) [4]. The latter generates data related to soil moisture and weather localized for use by 
specific farmers, drawing upon a large volume of public data for past years to construct risk models and project short term weather implications for timing of farmer decisions. The big challenge they curently face is similar to that of the other large companies invoved in data collection from farmers to then provide them specific mangement recommendations: How much data are farmers willing to provide free to the companies, who then provide recommendations back to the producers for a fee? Further, to what extent is the overall context of the individual farm operation able to be considred in creating management strategies based on this limited number of factors for soil moisture and weather?

Another example of private sector innovation utilizing big data generated from farm production equipment involves International Business Machines Corp.(IBM) plans to invest $\$ 3$ billion over the next four years to analyze data from sensor equipped devices and smartphones. In agriculture, IBM will create an alliance with the Weather Company to help farmers make more-timely and better decisions. The Weather Company already sells data to agriculture, but as weather sensors proliferate, more timely and localized data can be utilized to help farmers increase the precision of their predictions. They could even know when hail might impact their particular fields, for example [5].

\section{Climate-Smart Agriculture}

The burgeoning world population will increase demand for food, feed, fiber, and fuel from agricultural production. To meet the growing demand for agricultural products despite the impacts of climate change and the limited availability of additional agricultural lands, it will be critical to enhance productivity and resilience of the natural and agricultural ecosystem. Innovation in production practices will be needed, including developing adaptation and mitigation to offset climatic impacts. This will require transdisciplinary scientific collaboration which crosses disciplinary and sector boundaries to integrate research knowledge that brings the best science to agricultural and broader societal challenges in assuring food security worldwide. Supporting farm profitability with information about adapting agricultural practices and mitigation approaches to reducing the impacts of agriculture must include demonstrating benefits of incorporating climatesmart agriculture practices into public and private information delivery systems for farmers, enabling both better farm management strategies and public policies to implement environmentally sound farming practices [6].

Climate change is obviously too big and much more complex than can be dealt with by individual producers. Further, information about climate change tends to be broad rather than specific and therefore not relevant enough to inform individual decisions. While adaptation strategies informed by research to address climate impacts may help increase overall agricultural productivity, the strategies will not necessarily be very helpful to individual landowners. Place-based models driven by experts but transparent enough for producers to embrace and incorporating local knowledge and guidance may be needed to have the greatest impact for the individual landowners. Transdisciplinary research to develop models based on adaptation and transformation at either the farm or landscape level is needed. Examining multifunctional solutions for agronomic, ecological and socioeconomic challenges will be necessary. All of this requires strong analytics to sort out implications based on analysis of huge amounts of data to improve agricultural production while simultaneously minimizing environmental impacts.

One example of an approach to climate-smart agriculture is provided by the United States Department of Agriculture (USDA) Climate Hubs which provide a bridging function to support development of climate resilient agriculture. These Hubs facilitate communication and capacity building among organizations which can lead to creation of knowledge, tools and products based on informatics role in applied analytics that help create agricultural resilience by enabling farmers to make strategic decisions dealing with climate change. Examples from the United States, as well as from international university-based food security initiatives, provide insights into how climate smart agriculture may be developed locally in the context of international collaboration to address worldwide problems.

The USDA Climate Hubs research project uses building blocks for climate smart agriculture which link climate, crop and economic modeling with cutting-edge information technology. This allows the development of improved crop and economic models and next-generation climate impact projections for agriculture [6]. These building blocks include:

- Greenhouse Gas Reduction through Agricultural Carbon Enhancement Network (GRACEnet), an assessment of soil carbon sequestration and greenhouse gas mitigation by agricultural management. This requires large amounts of data to be collected and analyzed. Project details are available

at (http://www.ars.usda.gov/research/projects/projects. htm?ACCN_NO=411610).

- A whole farm and ranch carbon and greenhouse gas accounting system (COMET-FARM), again a major data gathering and analysis project. Project details are available at (www.nrcs.usda.gov/.../nrcs/main/national/climatec hangeCached).

- Agricultural Model Intercomparison and Improvement Project (AgMIP), to improve soil-crop models for evaluating effects of climate change and adaptations on production and natural resources. 
The European context is included through participation of Wageningen University, Netherlands. Project details are available at (http://ars.usda.gov/research/projects/projects.htm? ACCN_NO=427157).

Food security in the long run is the overriding issue which must be addressed and it will require technologically advanced agriculture capable of adapting to changing climatic conditions and weather patterns, just as it has done successfully over the years. The critical role of informatics and the ability to apply current analytics to the vast amounts of data generated by modern agricultural technology will be an important element of this effort to feed the burgeoning population and demand for improved diet quality. Farmer's knowledge base will be more comprehensive even for their own land and help them focus on the best combination of environmentally sound practices, efficient resource use, and productivity enhancing practices to help meet the food and feed needs of the future

\section{WeATHER, DROUGHT AND BIG DATA}

Over the past decade, the proportion of the United States facing moderate-to-severe drought has increased and remained at elevated levels for extended periods within that timeframe [7]. At the September 25 peak of the drought in $2012,65.45 \%$ of the continental United States was affected. Current drought conditions in California, a major producer of fresh and processed fruits and vegetables for the U.S. domestic and export markets, are at the extreme for even exceptional levels of drought severity and have been very high for the past several years [8]. This suggests that increased emphasis needs to be on creating long-term drought resilience as the drought in California has worsened steadily over the past five years. Developments in improving the capability of the agricultural sector to withstand the productivity and economic impacts of drought are becoming more critical and require capacity to monitor regional and national soil moisture content. The U.S. National Integrated Drought Information System (NIDIS) portal incorporates multiple drought indices providing quantitative assessment of various elements of drought severity including rainfall, evaporation, soil moisture, and streamflow based on land surface and hydrology [9]. This set of tools allows drought monitoring across the United States and facilitates development of regional drought monitoring systems. Preparing for drought and doing risk management planning at the regional or state level thus becomes possible, utilizing informatics capability to deal with the huge amounts of data generated.

Data on soil moisture is critical in order to assess significant factors related to planning and implementing drought impact mitigation strategies. Large amounts of measurements must be analyzed to develop localized strategies. Understanding drought conditions, the potential for floods, estimating crop yields, forecasting water supply, and creating hydrologic models are all important elements of dealing with the impacts of climate change induced weather variability reflected in drought situations. While there are many sources of information available, they differ highly in quality. In order to make useful projections, high quality point data from various sites must be compatible across the area of concern. Integrating that data with remote sensing at various scales allows scientists to develop models of drought impacts to evaluate risk management strategies and identify appropriate policy directives or private sector plans to deal with the risks.

Several efforts are underway in the United States to improve soil moisture data through remote sensing. Innovative efforts in soil moisture remote sensing include:

- Microwave and thermal infrared observations under the auspices of the National Oceanic and Atmospheric Administration (NOAA), a U.S. federal agency focused on the condition of the oceans and the atmosphere, changes in climate and weather understanding and prediction; and sharing knowledge and information with others to support resilient ecosystems. More information is available at: http://www.noaa.gov/about-noaa.html.

- University of Arizona Cosmic-ray Soil Moisture Observing System (COSMOS), a National Science Foundation (NSF) supported project to measure soil moisture on the horizontal scale of hectometers and depths of decimeters using cosmic-ray neutrons. Additional project information is available at: http://cosmos.hwr.arizona.edu/Docs/ProjectSummar y.pdf.

- NASA's Jet Propulsion Laboratory Soil Moisture Active/Passive (SMAP) satelliteis a remote sensing observatory which will map soil moisture and determine the freeze or thaw state of the area mapped, using radar and radiometer measurements. This will deliver high accuracy, high resolution global maps of the earth's soil moisture and freeze/thaw state. Project details are available at: http://smap.jpl.nasa.gov/observatory/overview/.

Scientists have a number of modeling efforts underway to utilize the best data available to accurately measure soil moisture at the working level scale, including a multimodal approach for North America. As part of a National Soil Moisture Network, a nationwide working group is addressing issues of scale and spatial distribution for networks, remote sensing platforms, and modeling efforts. The goal is to establish a nationwide product from existing soil moisture data. This will require integrating available soil moisture data types and sources to ascertain how the data could be used by researchers, policy agencies, and various sectors of the economy.

Significant effort is focused on improving the level of resolution models and remote sensing data which provide 
spatial coverage of soil moisture for the United States. Some of the innovative approaches discussed above are aimed at improving upon the current situation in which models generally only provide near-surface soil conditions since subsoil moisture is important in how well crops can adapt to dry conditions. Calibrating models to site specific measurements is a big challenge. Current efforts include a pilot soil moisture monitoring system for smaller regions integrating all available soil moisture data types. The goal is to assess our data they serve the needs of researchers governmental agencies various economic sectors. The pilot project leverages the plethora of existing networks and modeling efforts to identify best practices for calibration/validation and metadata characterization. The goal is to achieve near real-time soil moisture network implementation [9].

A global drought monitoring framework is also being developed to integrate continental and regional drought monitoring. Within the United States, NIDIS is integrating drought and drought impact information based on key indicators. This is expected to provide drought forecasts that are usable, reliable, and timely. It is anticipated to improve national coordination of soil moisture monitoring [8]. The development of this integrated framework requires a focus on capacity building and improving decisions based on big data. This can increase accuracy in measuring the heterogeneity, scale, timeliness, and complexity involved in monitoring and predicting the impacts of climate change on agricultural productivity. Success of these efforts has implications for food security throughout the world.

\section{SUPPLY CHAIN FOOD SAFETY}

Another critical element of food security is food safety throughout the food processing and distribution supply chain. There are an estimated 48 million U.S. foodborne illnesses each year and one estimate puts costs associated with foodborne illness at $\$ 78$ billion per year in the United States. This is a conservative estimate which takes into account only some of the potential long-term health outcomes in addition to immediate costs for treatment and lost income impacts on individuals involved [10]. However, the causative pathogen and the producer or food company are rarely linked in these incidents. Therefore, private market incentives to assure food safety are weak. One approach to increase economic incentives and accountability to control foodborne pathogens would be for the United States, or any country/ government to establish a policy laying out a set of actions or activities required of food processors and handlers in the supply chain. Two important components of such a policy would be to require repetitive testing from farm gate to final consumer, or farm to fork; and to create a nationwide database linking pathogens to specific food products and to the companies that supply contaminated foods [11].
This means that setting priorities for interventions to control pathogens is needed and potentially of significant value. Risk assessment and cost/benefit analysis can be used to evaluate pathogen interventions in the food supply chain, then used to set priorities and targets for specific foodborne illnesses. A major challenge is to increase available information through public health databases and liability databases, tying specific foodborne illnesses to specific food products and the producers or companies supplying them, as noted previously [12, 13$]$.

The first point with potential to keep pathogen contamination out of the food supply chain is in the production fields. This is especially important with fresh produce which receives minimal processing beyond the farm gate. A project is currently underway to create a GISbased online tool to identify specific locations within fields of produce farms where foodborne pathogens are present at an elevated level. This will allow growers to focus food safety efforts based on risk based strategies. They will be able to implement more informed management decisions and science-based strategies to limit produce contamination in the field. The task will be made easier by heat maps pinpointing relative risk levels in each field [14].

At the macro level, public and private sector networks to analyze big data and apply it to tracing causes of food safety outbreaks to eliminate or mitigate them increasingly exists within countries and in international contexts. In the United States, the 2011 FDA Food Safety Modernization has created incentives for the private sector to adopt HACCP practices which include properly cleaning processing plants and keeping foods well refrigerated including during transportation. Food producers and transporters now employ sensor-based technologies and big data analytics to strengthen the food safety characteristics of their supply chains. Widespread use of personal devices, in-plant scanning equipment, detailed shipment tracking, and consumer purchase monitoring by retailers produces the big data that enhances traceability from the producer through to consumers [15].

Perhaps the most important food safety concerns currently exist in rapidly emerging economies where growing demand for animal-source foods and vegetables which are among the riskiest in terms of food safety, as well as rapidly intensifying agriculture to meet the rising demand for foods that occur in a setting where the governance systems lag the rapid growth. Food safety and prevention of disease from agricultural production and marketing systems are global public goods. To protect the integrity of the global food system necessitates particular attention to the role of and mechanisms for assuring food safety in emerging economies supplying foods for export. This requires international cooperation and investment in safer foods and agriculture, both by the international community and national governments [15]. 
An example of a more micro-level development in big data application to improve food safety is a machine using optical-scanning, laser-sensor technology developed by Purdue University in the United States that scans bacteria colonies and can pinpoint eight specific variations in Salmonella. It can also identify other major food safety pathogens, including Escherichia coli, Vibrio, Listeria, Bacillus and other foodborne pathogens at greater than $95 \%$ accuracy. A major advantage is its ability to produce results within 24 hours, one third of the time required by existing technologies. It is anticipated to provide an effective preliminary screening tool that is inexpensive to implement as well as fast and efficient. These characteristics should appeal to food processing sector and this technology may be expected to be rapidly adopted [16].

\section{DATA OWNERSHIP, CONTROL AND PRIVACY}

Some farmers are concerned about providing to large companies detailed information about their farm operations. This has been a contentious issue for the past several years. . The concern among farmers largely centers on privacy and information concerns, given the increasing number of data sensors installed in equipment used for crop planting, production and harvesting. An agreement was reached in November 2014 between the large chemical and farm equipment companies and farmers represented by the major farmer organizations. It specifies that farmers own the data generated from their operations and the companies collecting the data must disclose how it may be used and types of third parties who may obtain access to it $[17,18]$. This agreement is expected to accelerate the private sector investment in moving to the next generation of precision agricultural data technology to increase efficiency of food production while protecting the environment, further boosting farm productivity across the United States.

These concerns about ownership and control, especially affecting potential privacy issues of making it possible for rival organizations to deduce who is providing the data will likely need attention throughout the food supply chain, as well as in access to data for researchers relying on it to undertake analyses which provide information for decision makers in the public and private sectors. These concerns are a topic of widespread concern within the private and public sectors in the United States, European Union and worldwide. The extent to which society is able to balance accuracy and privacy of research protocols will directly impact the extent to which big data benefits everyone from advances in science rather than only those private interests controlling voluminous stores of sensitive information [19].

The widely used current set of privacy protocols originated in the United States 40 years ago as Fair Information Practices (FIPs) and were revised by the Organization of Economic Cooperation and Development (OECD) in Paris. Subsequently, many national and international privacy regulations have spun off from those standards created in response to computerized data systems making data readily accessible. While those notice and consent regulations protected individuals by assuring that there were no secret data-collection systems and participation in the data collection was voluntary, they are not well suited to today's system compiling huge amounts of data from various automated sources. Scientific and political opinion is rapidly coalescing around the need to control how the data is exploited, rather than how it is initially collected and used. Policy must establish controls over data usage and then automated controls will need to be developed around precise usage rules, which will also provide transparency [20].

Of course, it is not just farmers who are concerned about privacy and loss of control over data and information about their business operations. This is likely to be an issue along with entire food supply chain, particularly when it comes to dealing with food safety. Among recommendations likely be considered in that realm is building a database to publicly link food outbreaks to specific firms. The rationale is that by quickly identifying the source of food safety incidents and publicizing businesses linked thereto, it would enable some number of consumers to avoid foodborne illness. Further, these issues will not be confined to the food system, as they are obviously critical to healthcare and financial transactions among many other segments of the economy. It will affect academic access to research data, business transactions, and governmental agencies as well as citizens. Those of you directly involved in creating protocols for big-data collection and use will be an integral part of this process.

\section{LOOKING AHEAD}

Developing policy options for research on climate change impacts and solutions, the need for funding data collection, and the necessity of partnering broadly to deal with the scope of challenges involved are all integral to improving the food supply chain to ensure food security for the future. Biological research to facilitate adaptation and mitigation must be accompanied by hydrologic, economic, and other related research to develop a robust policy environment. More sophisticated models incorporating better data will be required for developing localized solutions and policies to facilitate actions needed. This will undoubtedly require better university and private sector partnerships including international collaboration due to the global nature of the problem [21].

Several issues deserve particular attention in the policy arena as well as by the data gathering and analytics communities. How can big data access be provided across the range of food producers and geographies to facilitate good production decisions worldwide to produce food security for over 9 billion people by 2050? How can the large numbers of very small farmers who produce a large portion of food in the poorest economies be helped by 
access to the power of big data, and how can that access be provided to increase their efficiency and productivity? Will it be necessary for the private sector to provide free access to farm management solutions they create to obtain continued participation by farmers and academics working in the arena? What data standards and publication protocols will need to be established regarding anonimization and aggregation before publication of information derived from the analysis and application of big data in the agriculutral and food sectors?

Dealing with climate related drought and water availability issues is a more national and regional issue, but sharing experience among countries through international statistical structures can increase efficiency in addressing these issues. Similarly, food safety issues as part of the food security equation are local, regional or national in many cases. However, in today's globalized economy collaboration is necessary to assure food safety internationally. Technology innovations offer promise for improved avoidance of foodborne illnesses, tracing to the source those that occur, and reducing the number of illnesses and deaths associated with foodborne pathogens. Speeding up detection of contamination sources and preventing further spread of illness in developing as well as in developed countries will greatly reduce human suffering and costs associated with foodborne illnesses. Informatics has much to contribute to this outcome by developing protocols for rapid analysis of big data to avoid or mitigate food safety incidents.

The application of big data to all these issues within the supply chain carries the potential to raise ownership, control and privacy issues around data. It will take the scientific community, private sector and government representatives working together to develop sound policies to mitigate concerns and impacts of data use that goes beyond the intended purposes for which the data was collected.

There are many challenges which will need to be overcome in using big data to improve the food security worldwide. The informatics community is in a prime position to develop robust and productive approaches to assuring that society receives the greatest benefits from food security that can be improved by insightful application of big data.

\section{REFERENCES}

[1] W. J. Armbruster and M. C. Ahearn, "Changing structure and organization of US agriculture," in Encyclopedia of Agriculture and Food Systems, Vol. 2, N. Van Alfen, Editor-in-chief. San Diego: Elsevier, 2014, pp. 201-219.
[2] C. Fraisse, "AgroClimate: Climate tools and information for the southeast USA," USDA 2015 Agricultural Outlook Forum, Arlington, VA: February 19, 2015.

[3] J. L. Hatfield, "Remote sensing and risk management tools," USDA 2015 Agricultural Outlook Forum, Arlington, VA: February 19, 2015.

[4] M. Specter, "Climate by numbers: Can a tech firm help farmers survive global warming?" Annals of Science, November 11, 2013.

[5] D. Clark, "IBM to invest $\$ 3$ billion to build sensor-data business." The Wall Street Journal, March 31, 2015, page B4.

[6] K. Steenwerth, "Defining climate-smart agriculture," USDA 2015 Agricultural Outlook Forum, Arlington, VA: February 20, 2015.

[7] R. S. Pulwarty, "The national integrated drought information system: big data and bigger questions," USDA 2015 Agricultural Outlook Forum, Arlington, VA: February 20, 2015.

[8] R. Velasco, "Dealing with drought at the regional level: the California crisis," USDA 2015 Agricultural Outlook Forum, Arlington, VA: February 20, 2015.

[9] M. L. Strobel, "Identifying New Opportunities: Developing a Coordinated National Soil Moisture Network," USDA 2015 Agricultural Outlook Forum, Arlington, VA: February 20, 2015.

[10] R. Scharff, "Economic burden from health losses due to foodborne illness in US", J Food Protection, vol. 75 (1): 123-131, 2008.

[11] T. Roberts, "'Free riders' and weak economic incentives to control foodborne pathogens," unpublished, January 20, 2015.

[12] T. Roberts, "Lack of information is the root of US foodborne illness risk," Choices, 2nd Quarter, vol 28(2), 2013. Available at: www.choicesmagazine.org/magazine/pdf/cmsarticle 300.pdf).

[13] M. Wiedman, "Validation of geospatial algorithms to predict the prevalence and persistence of pathogens in produce fields to improve GAPs," The Center for Produce Safety, Davis, CA: January 1, 2014. Available at

http://www.Centerforproducesafety.org/researchproject/371/awards/Validat ion_of_geospatial_algorithms_to_predict_the prevalence...

[14] W. J. Armbruster and M. M. MacDonell, "Informatics to support international food safety," Proceedings of the 28th EnvironInfo 2014 Conference, Oldenburg, Germany: September 10-12, 2014.

[15] D. Grace and J. McDermott, "Reducing and managing food scares," in 2014-2015 Global Food Policy Report, Washington, DC: International Food Policy Research Institute, March 18, 2015, pp 41-50.

[16] N. van Hoose, "Laser tool speeds up detection of Salmonella in food," Purdue Alumnus, May/June, 2015. Available at: www.Purdue alumni.org.

[17] D.L. Goroff, "Balancing privacy versus accuracy in research protocols", Science vol. 347 (6221), p. 479, 30 January, 2015.

[18] American Farm Bureau Federation, "Farmers, Agriculture Technology Providers Reach Agreement on Big Data Privacy and Security Principles Expected to Accelerate Technology Adoption", The Voice of Agriculture, Kansas City, Missouri: November 13, 2014. Available at: http://www.fb.org/index.php?action=newsroom.news article\&id=188.

[19] American Farm Bureau Federation, "Privacy and security principles for farm data", Washington, DC, November 13, 2014. Available at: http://www.fb.org.

[20] S. Landau, Control use of data to protect privacy, Science vol. 347(6221) pp. 504-506, 30 January, 2015.

[21] G. Nelson, "Supporting profitability with climate-smart agriculture," USDA 2015 Agricultural Outlook Forum, Arlington, VA: February 19, 2015 